\title{
Clinical Performance of Samfilcon A, a Unique Silicone Hydrogel Lens, on a 7-Day Extended Wear Basis
}

This article was published in the following Dove Press journal: Clinical Ophthalmology

\author{
William Reindel ${ }^{1}$ \\ Gary Mosehauer' \\ Marjorie Rah' \\ Howard Proskin (1D) \\ Robert Steffen' \\ 'Vision Care, Bausch \& Lomb \\ Incorporated, Rochester, NY, USA; \\ ${ }^{2}$ Howard M. Proskin \& Associates, \\ Rochester, NY, USA
}

Correspondence: Marjorie Rah

Vision Care, Bausch \& Lomb

Incorporated, Rochester, NY, USA

$\mathrm{Tel}+$ I 585-4I3-6397

Email Marjorie.Rah@bausch.com
Purpose: The objective of this study was to evaluate and compare the clinical performance of samfilcon A, a unique, polyvinylpyrrolidone (PVP)-containing, silicone hydrogel contact lens with that of the balafilcon A silicone hydrogel contact lens when worn on a 7-day extended wear basis.

Subjects and Methods: A total of 669 subjects completed this 12-month, controlled, parallel group, masked, randomized study; of these, 340 wore samfilcon A lenses and 329 balafilcon A lenses. Subjects wore their respective assigned lenses bilaterally on a 7-day extended wear basis. On the seventh night of each wearing week, lenses were removed, cleaned, and disinfected using Biotrue multi-purpose solution (MPS), then re-inserted the following morning. Lenses were replaced with new lenses monthly. At each follow-up visit, investigators completed a slit lamp evaluation, and subjects rated lenses based upon a predefined set of performance criteria.

Results: The samfilcon A lens performed comparably to the balafilcon A lens in terms of most graded and ungraded slit lamp findings, differing significantly only for corneal staining Grade 2 or greater, which favored samfilcon A, and anterior segment abnormalities, which favored balafilcon A. Subjects rated both lenses highly when queried about various lens-wearing characteristics. When comparing the number of subjects with findings on either eye on at least one follow-up visit, the two lenses were comparable in many aspects but favored samfilcon A with respect to cleanliness upon removal, overall comfort, comfort at end of day, dryness, vision, vision in low light, vision at end of day, and overall impression (all $\mathrm{p}<0.05$ ).

Conclusion: While subjects rated both highly, samfilcon A lenses worn for 7-day extended wear and replaced on a monthly basis performed comparably to or better than balafilcon A lenses when worn for the same 7-day wear time and replacement cycle.

Keywords: PVP, contact lens, extended wear

\section{Introduction}

Today's contact lens wearers have more lens options from which to choose than ever. ${ }^{1}$ Daily-disposable lenses are designed to be worn for a single day, then disposed of upon removal, while planned-replacement lenses are designed to be worn daily and cleaned and disinfected nightly, then disposed of after a maximum number of wearing days. Extended-wear lenses are designed to be worn for up to a maximum number of days between cleaning and disinfection, and disposed of after a maximum number of wearing days.

Four leading, currently marketed soft lenses are indicated for daily wear or extended wear for up to 7 days between removals for cleaning and disinfection or 
disposal as recommended by the eye care practitioner, these being senofilcon A (Acuvue Oasys, Johnson \& Johnson Vision Care, Jacksonville, FL), ${ }^{2}$ samfilcon A (Bausch + Lomb ULTRA, Bausch \& Lomb Incorporated, Rochester, NY), ${ }^{3}$ comfilcon A (Biofinity, CooperVision, Pleasanton, CA) ${ }^{4}$ and lotrafilcon B (Air Optix Aqua, Alcon, Ft. Worth, TX). ${ }^{5}$ Two currently marketed lenses are approved for daily wear or extended wear up to 30 days between removals, these being balafilcon A (PureVision, Bausch \& Lomb Incorporated) ${ }^{6}$ and lotrafilcon A (Air Optix Night and Day, Alcon). ${ }^{7}$ Both 30-day extended wear lenses were initially approved for daily wear or weekly extended wear but achieved monthly extended wear indications after the appropriate clinical trials.

Modern contact lens material development is complex as lens materials must withstand a range of user conditions influenced by environmental factors (eg, low relative humidity), ${ }^{8,9}$ demanding visual tasks (eg, increased attention to digital devices), ${ }^{10,11}$ digital screen viewing that affects both blink dynamics and tear film characteristics, ${ }^{11-13}$ individual characteristics (eg, tear film anomalies), ${ }^{14}$ and lens care factors (eg, rub/rinse manipulation). ${ }^{15}$ Advancements in contact lens materials and design can help sustain comfort and vision across recommended replacement cycles. Manufacturers often include wetting agents in lens formulations to promote water retention, most notably phosphorylcholine $(\mathrm{PC})^{16}$ and polyvinylpyrrolidone (PVP). ${ }^{17}$ Such agents are desirable as contact lens components due to their waterattracting properties. Samfilcon A, a unique, PVP-containing, silicone hydrogel lens, ${ }^{3}$ was designed to retain moisture and maintain a persistent, smooth tear film surface. ${ }^{18}$ Synthesis of the samfilcon A lens polymer was described in detail previously. ${ }^{19}$ Briefly, samfilcon A is a silicone hydrogel fabricated from a proprietary siloxane macromer and NVP monomer. The lens material was designed to polymerize in two kinetic phases, with the rapid formation of a silicone network, followed by the later formation of a PVP internal wetting agent. The material and process are designed to deliver a smooth, durable surface for spreading the tear film, reducing friction, and providing precision optics. Maintaining the key performance characteristics throughout the recommended replacement cycle plays an important role in wearer satisfaction.

The samfilcon A lens performs well clinically when worn on a daily wear basis with monthly lens replacement.
In one study, 341 patients rated samfilcon A better than their respective habitual lenses in terms of comfort, vision, and on-eye lens characteristics after a 2-week period of daily wear. ${ }^{20}$ In the same study, 226 digital device users (3 hours or more daily use) that wore their respective habitual lenses at least 12 hours daily and self-reported dryness and/or blurry/fluctuating vision with contact lens wear, similarly rated samfilcon A lenses superior to their habitual lenses in terms of comfort, dryness, redness, vision, and on-eye lens characteristics after a 2-week period of daily wear. ${ }^{21}$ In a different study, high samfilcon A lens ratings persisted across the manufacturer-recommended monthly replacement schedule, as well as over 3 months of daily wear, with lenses cleaned and disinfected nightly and replaced monthly. ${ }^{22}$ As FDA only recently approved samfilcon A lenses for 7-day extended wear in the United States (US), ${ }^{23}$ it is of interest to know how these lenses perform when worn on an extended-wear basis relative to control lenses. Therefore, the objective of the present study was to evaluate the clinical performance of samfilcon $\mathrm{A}$ as a 7-day extended wear lens with monthly replacement, and compare to balafilcon $\mathrm{A}^{24}$ an approved 30-day extended wear lens, worn in this study on the same basis as samfilcon A.

\section{Materials and Methods Subjects and Lenses}

The Quorum Institutional Review Board approved the study protocol prior to subject recruitment, and the trial was conducted in accordance with the Declaration of Helsinki. A total of 36 investigators across 36 investigative sites in the US participated in this 12-month randomized, parallel, investigator-masked study. All potential study subjects provided informed consent prior to the determination of eligibility. Eligible subjects were myopic, adapted planned-replacement, soft contact lens wearers aged 18 to 40 on the date of informed consent. All subjects required lens correction from -0.50 diopters (D) to $-6.00 \mathrm{D}$ in each eye and were correctable through spherocylindrical refraction to 32 letters (0.3 logMAR visual acuity) or better (distance, high contrast) in each eye, had clear central corneas, and were free of any anterior segment disorders, any graded corneal infiltrate, and any other graded slit lamp finding of Grade 2 or greater at screening.

At the baseline screening visit, eligible subjects were randomized to receive either samfilcon A (test) or 
balafilcon A contact lenses (control) and were dispensed their respective assigned lenses. Subjects wore lenses bilaterally on a 7-day/6-night extended wear basis with monthly replacement. On the seventh night of each week, lenses were removed, cleaned, and disinfected using Biotrue multi-purpose solution (MPS) (Bausch \& Lomb Incorporated $)^{25}$ according to the manufacturer's instructions per the product package insert, then reinserted the following morning.

Subjects returned to the clinic for assessment 1 day, 1 week, 1 month, 3 months, 6 months, 9 months, and 12 months after the initial screening/dispensing visit. In addition, subjects returned to the clinic for unscheduled visits whenever they experienced problems with their lenses. Lenses were provided at the Screening/Dispensing, 3-Month, 6-Month, and 9-Month Follow-Up Visits to maintain a monthly replacement schedule.

\section{Qualitative and Quantitative Assessment}

At each scheduled and unscheduled follow-up visit, investigators completed a slit lamp evaluation and examined subjects for indications of adverse events (AEs). Slit lamp signs of epithelial edema, epithelial microcysts, limbal injection, bulbar injection, corneal infiltrates, corneal neovascularization, upper lid tarsal conjunctival abnormalities, and corneal staining were graded using an ordinal, text-based scale from which numeric grades in integer steps were assigned, 0 (no finding), 1 (trace), 2 (mild), 3 (moderate) and 4 (severe). Fluorescein staining grades were computed as the maximum grade taken within each of five different corneal locations (central, inferior, nasal, superior, and temporal). Other ungraded slit lamp findings, including new corneal scars, corneal striae, conjunctivitis, external adnexa abnormalities, and other anterior segment abnormalities, were marked as either present or absent for each eye.

Subjects rated lenses based upon comfort, vision, lowlight vision, and cleanliness using a 0 to 100 scale, with 0 denoting the least favorable and 100 denoting the most favorable rating. For context, descriptors were added to the rating scale for each category. As an example, the "overall comfort" category designated 100 as "excellent; cannot be felt," 80 as "very comfortable; just felt occasionally," 60 as "comfortable; noticeable but not irritating," 40 as "uncomfortable; irritating or annoying," 20 as "very uncomfortable; very irritating or annoying," and 0 as "painful; difficult to wear lenses."

\section{Statistical Analysis}

The two primary objectives of the statistical analyses were 1. to provide a high-level, descriptive overview of the data, and 2. to provide an overview comparison between the treatment groups with respect to the occurrence of untoward events (as defined below) observed during each subject's follow-up visits, including unscheduled visits. The manner by which these were addressed is described below. A significance level of $\alpha=0.05$ was utilized for all reported statistical tests of hypotheses.

\section{Graded Slit Lamp Findings}

To provide a descriptive overview of the graded slit lamp findings, the distribution of scores for each graded slit lamp parameter tallied all individual eye-wise scores (both OD and OS) for every subject at every follow-up assessment. No statistical analysis was performed as these data do not represent independent sampling units. To investigate untoward events for each graded slit lamp parameter, each subject was categorized according to whether or not a finding of grade 2 or higher was recorded on either eye (OD or OS) at any follow-up visit. The number and proportion of subjects for whom such findings were observed was tabulated, and the proportions were compared between lens groups using Fisher's exact test.

\section{Ungraded Slit Lamp Findings}

For all ungraded slit lamp parameters, the comparison of untoward events also serves as a useful overview. Each subject was categorized according to whether or not a positive finding was recorded on either eye (OD or OS) at any follow-up visit. The number and proportion of subjects for whom such findings were observed were tabulated, and the proportions were compared between lens groups using Fisher's exact test.

\section{Symptoms/Complaints Scores}

For each symptom/complaint, the descriptive overview was based upon the overall mean score for each subject, which was determined as follows: (1) the average score across OD and OS was calculated for each visit; (2) the mean of these per-visit averages was then calculated across all follow-up visits at which the subject presented data for the symptom. A summary of the subject-wise overall mean scores was tabulated. No statistical analysis was performed as these data do not represent independent sampling units.

To investigate untoward events, each subject was categorized according to whether or not an unfavorable finding 
(score $<50$ ) was recorded on either eye (OD or OS) at any follow-up visit. The number and proportion of subjects for whom such unfavorable findings were observed was tabulated, and the proportions were compared between lens groups using Fisher's exact test.

\section{Results}

\section{Subject Demographics}

Demographics of dispensed subjects are shown in Table 1. The samfilcon A and balafilcon A subgroups did not differ with respect to age, gender, or race (all $p \geq 0.05$ ).

All 816 enrolled subjects (406 and 410 randomized to samfilcon A and balafilcon A, respectively) were given a baseline examination. One eligible subject randomized to samfilcon A was not dispensed lenses, and ten subjects (two randomized to samfilcon $\mathrm{A}$ and eight to balafilcon A) were ultimately determined ineligible to complete the study and disenrolled (Figure 1). As such, statistical comparisons were made using data from a nominal population of 403 and 402 subjects, randomized to samfilcon A and balafilcon A, respectively, including all scheduled visits, as well as 267 unscheduled visits (142 for the samfilcon A group, and 125 for the balafilcon A group). The actual number of observations for each group was up to 3 less than the nominal number for some measured parameters due to missing data from the investigators and was used in all hypothesis testing calculations.

Of 805 subjects who were dispensed lenses, 669 (83.1\%) completed the study. Of those that did not complete the study, only 11 eyes $(0.01 \%)$ were discontinued due to an
Table I Demographics of Eligible Dispensed Subjects

\begin{tabular}{|l|l|l|l|}
\hline Characteristic & $\begin{array}{l}\text { Samfilcon A } \\
(\mathbf{n}=\mathbf{4 0 3})\end{array}$ & $\begin{array}{l}\text { Balafilcon A } \\
(\mathbf{n}=\mathbf{4 0 2})\end{array}$ & p-value \\
\hline $\begin{array}{l}\text { Age (years) } \\
\text { Mean } \pm \text { SD }\end{array}$ & $29.8 \pm 6.2$ & $29.2 \pm 6.1$ & $0.17^{\#}$ \\
\hline $\begin{array}{l}\text { Gender: } n(\%) \\
\text { Female }\end{array}$ & $263(65.3)$ & $265(65.9)$ & $0.84^{\S}$ \\
Male & $140(34.7)$ & $137(34.1)$ & \\
\hline Race: $n$ (\%) & $326(80.9)$ & $328(81.6)$ & $0.63^{\S}$ \\
White & $33(8.2)$ & $30(7.5)$ & \\
Black/African American & $15(3.7)$ & $19(4.7)$ & \\
Asian & $25(6.2)$ & $18(4.5)$ & $7(1.7)$ \\
Other & $4(1.0)$ & 7 & \\
Refused to Answer & & & \\
\hline
\end{tabular}

Notes: ${ }^{\S}$ Between-group p-value comparing category proportions based on chisquared test. The test for race excluded subjects that refused to answer. ${ }^{\#}$ Betweengroup p-value comparing mean scores based on two-sample $t$-test.

$\mathrm{AE}$ (all non-serious), while 58 were lost to follow-up and 80 voluntarily discontinued the study. When an AE occurred in one eye only, the subject and his/her contralateral eye was also discontinued from the study.

The distribution of graded slit lamp findings is summarized in Table 2. Follow-up scores obtained on both right and left eyes of all subjects for each finding, lens group, and lens grade are reported. Noteworthy is how few occurrences of greater than Grade 2 slit lamp findings were observed, with only four Grade 4 findings (one corneal infiltrate and three upper lid tarsal conjunctival abnormalities), and few Grade 3 findings (18 upper lid tarsal conjunctival abnormalities, 14

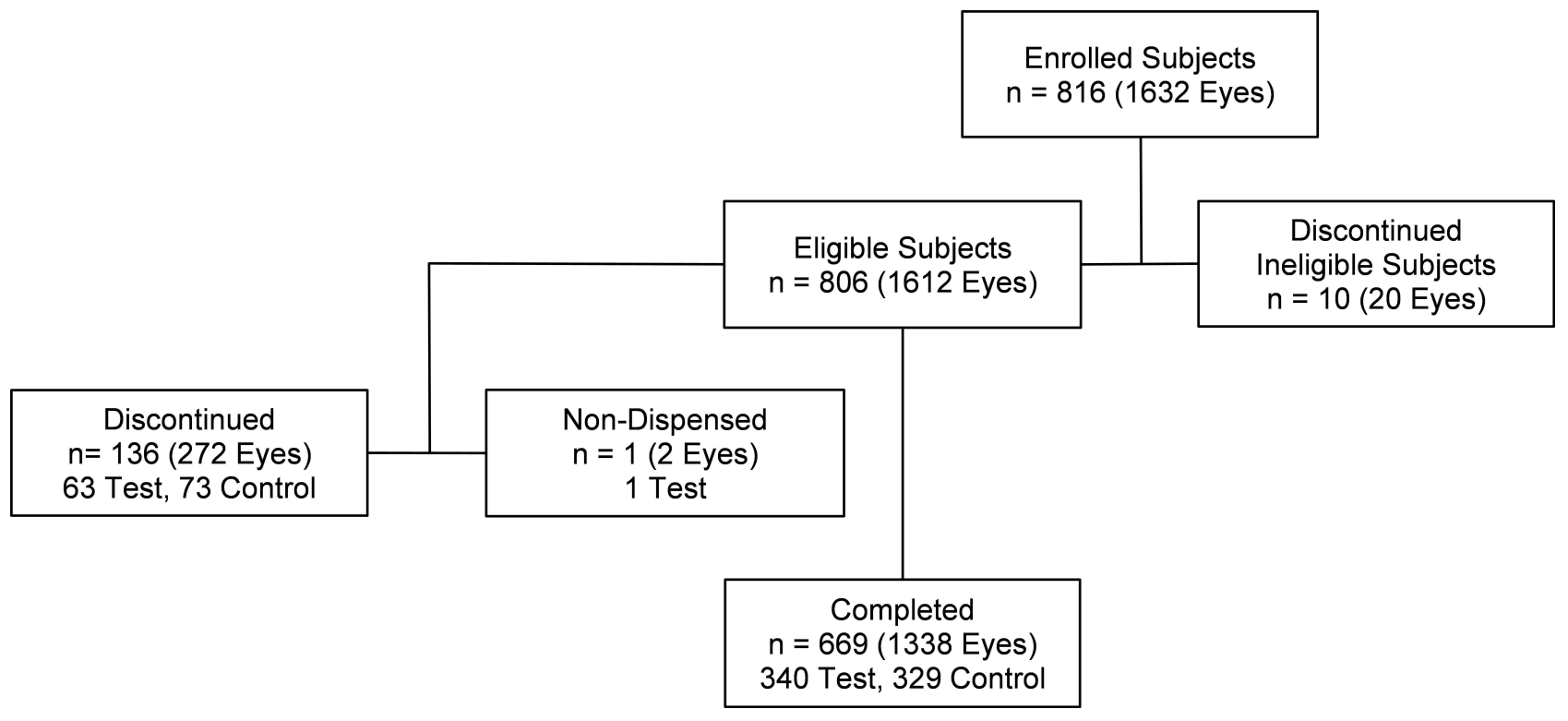

Figure I Subject enrollment flow chart. 
Table 2 Graded Slit Lamp Findings Over All Follow-Up Visits

\begin{tabular}{|c|c|c|c|c|c|c|}
\hline \multirow[t]{2}{*}{ Finding } & \multirow[t]{2}{*}{ Lens Group } & Grade 0 & Grade I & Grade 2 & Grade 3 & Grade 4 \\
\hline & & \multicolumn{5}{|c|}{ Number of Eyes (\% of Eyes) } \\
\hline \multirow[t]{2}{*}{ Epithelial Edema } & Samfilcon A & $5470(99.5)$ & $19(0.35)$ & $6(0.11)$ & $0(0)$ & $0(0)$ \\
\hline & Balafilcon A & $5358(99.4)$ & $28(0.52)$ & $5(0.09)$ & $0(0)$ & $0(0)$ \\
\hline \multirow[t]{2}{*}{ Epithelial Microcysts } & Samfilcon A & 5481 (99.8) & $14(0.25)$ & $0(0)$ & $0(0)$ & $0(0)$ \\
\hline & Balafilcon A & 5371 (99.6) & $19(0.35)$ & I (0.02) & $0(0)$ & $0(0)$ \\
\hline \multirow[t]{2}{*}{ Limbal Injection } & Samfilcon A & $5154(93.8)$ & $314(5.7)$ & $20(0.36)$ & $7(0.13)$ & $0(0)$ \\
\hline & Balafilcon A & $5027(93.3)$ & $345(6.4)$ & $12(0.22)$ & $7(0.13)$ & $0(0)$ \\
\hline \multirow[t]{2}{*}{ Bulbar Injection } & Samfilcon A & $5019(91.3)$ & $434(7.9)$ & $34(0.62)$ & $8(0.15)$ & $0(0)$ \\
\hline & Balafilcon A & $4922(91.3)$ & $437(8.1)$ & $26(0.48)$ & $6(0.11)$ & $0(0)$ \\
\hline \multirow[t]{2}{*}{ Corneal Infiltrates } & Samfilcon A & $5459(99.3)$ & $19(0.35)$ & $13(0.24)$ & $3(0.05)$ & $\mathrm{I}(0.02)$ \\
\hline & Balafilcon A & $5378(99.8)$ & $7(0.13)$ & $4(0.07)$ & $2(0.04)$ & $0(0)$ \\
\hline \multirow[t]{2}{*}{ Corneal Neovascularization } & Samfilcon A & $5349(97.3)$ & $146(2.7)$ & $0(0)$ & $0(0)$ & $0(0)$ \\
\hline & Balafilcon A & $5229(97.0)$ & $162(3.0)$ & $0(0)$ & $0(0)$ & $0(0)$ \\
\hline \multirow[t]{2}{*}{ Upper Lid Tarsal Conjunctival Abnormalities } & Samfilcon A & $4197(76.4)$ & $1204(21.9)$ & $80(1.46)$ & $12(0.22)$ & $2(0.04)$ \\
\hline & Balafilcon A & $3945(73.2)$ & $1336(24.8)$ & $103(1.91)$ & $6(0.11)$ & $\mathrm{I}(0.02)$ \\
\hline \multirow[t]{2}{*}{ Corneal Staining } & Samfilcon A & $3781(79.9)$ & $918(19.4)$ & $30(0.63)$ & $6(0.13)$ & $0(0)$ \\
\hline & Balafilcon A & $3523(76.0)$ & $1021(22.0)$ & $89(1.92)$ & $3(0.06)$ & $0(0)$ \\
\hline
\end{tabular}

Note: Distribution is based on all individual follow-up eye visits (including both OD and OS) for all subjects.

limbal injection, 14 bulbar injection, 5 corneal infiltrates, and 9 corneal staining).

The number of subjects with Grade 2 or greater finding on either eye on at least one follow-up visit appears in Table 3 for both lenses. The two lenses were comparable in all graded conditions except for corneal staining, which favored samfilcon A $(p<0.05)$.

The number of subjects with ungraded slit lamp findings present on either eye on at least one follow-up visit appears in Table 4 for both lenses. The two lenses were comparable in most aspects but favored balafilcon $\mathrm{A}(\mathrm{p}<0.05)$ with respect to other anterior segment abnormalities, the majority of which for both groups were inconsequential conjunctival findings such as nevi, pinguecula, pterygium, etc.

\section{Lens Performance}

Subjects rated symptoms/complaints at each visit (rating scale 0 to 100 , with 100 being the most favorable score). A descriptive summary of the subject-wise overall mean scores for all symptom/complaint parameters over all follow-up visits is shown in Table 5. However, both lenses scored highly, with most mean scores exceeding 90 , and all mean scores exceeding 80 .

The number of subjects with unfavorable finding on either eye on at least one follow-up visit appears in Table 6 for both lenses.
The two lenses were comparable in many aspects but favored samfilcon A with respect to cleanliness upon removal, overall comfort, comfort at end of day, dryness, vision, vision in low light, vision at end of day, and overall impression (all $\mathrm{p}<0.05$ ).

Table 3 Subject-Wise Slit Lamp Findings Grade 2 or Greater Over All Follow-Up Visits

\begin{tabular}{|c|c|c|c|}
\hline \multirow[t]{2}{*}{ Finding } & \multicolumn{2}{|c|}{$\begin{array}{l}\text { Number (Percent) of } \\
\text { Subjects with Grade } 2 \text { or } \\
\text { Greater Finding on Either } \\
\text { Eye on at Least One } \\
\text { Follow-Up Visit }\end{array}$} & \multirow[t]{2}{*}{$\begin{array}{l}t \\
\text { *p-value }\end{array}$} \\
\hline & $\begin{array}{l}\text { Samfilcon } \\
\text { A }(n=402)\end{array}$ & $\begin{array}{l}\text { Balafilcon } \\
\text { A }(n=400)\end{array}$ & \\
\hline Epithelial Edema & $4(1.0)$ & $4(1.0)$ & $>0.99$ \\
\hline Epithelial Microcysts & $0(0)$ & $\mathrm{I}(0.3)$ & 0.50 \\
\hline Limbal Injection & $21(5.2)$ & $14(3.5)$ & 0.30 \\
\hline Bulbar Injection & $33(8.2)$ & $23(5.8)$ & 0.21 \\
\hline Corneal Infiltrates & $12(3.0)$ & $4(1.0)$ & 0.07 \\
\hline Corneal Neovascularization & $0(0)$ & $0(0)$ & NA \\
\hline $\begin{array}{l}\text { Upper Lid Tarsal } \\
\text { Conjunctival Abnormalities }\end{array}$ & $27(6.7)$ & $39(9.8)$ & 0.12 \\
\hline Corneal Staining ${ }^{\#}$ & $27(6.7)$ & $46(11.6)$ & $0.02 *$ \\
\hline
\end{tabular}

Notes: 'Between-treatment $p$-value comparing proportions based on Fisher's exact test. ${ }^{\#}=40 \mathrm{I}$ for samfilcon $A$ and 397 for balafilcon $A$ for this parameter only. *Difference deemed significant at $\mathrm{p}<0.05$.

Abbreviation: NA, not applicable. 
Table 4 Subject-Wise Ungraded Slit Lamp Findings Over All Follow-Up Visits

\begin{tabular}{|c|c|c|c|}
\hline \multirow[t]{2}{*}{ Finding } & \multicolumn{2}{|c|}{$\begin{array}{l}\text { Number (Percent) of } \\
\text { Subjects with Finding on } \\
\text { Either Eye on At Least } \\
\text { One Follow-up Visit }\end{array}$} & \multirow[t]{2}{*}{ t**p-value } \\
\hline & $\begin{array}{l}\text { Samfilcon } \\
\text { A }(n=402)\end{array}$ & $\begin{array}{l}\text { Balafilcon } \\
A(n=400)\end{array}$ & \\
\hline New Corneal Scars & $8(2.0)$ & $2(0.5)$ & 0.11 \\
\hline Corneal Striae & $2(0.5)$ & $0(0)$ & 0.50 \\
\hline Conjunctivitis & $18(4.5)$ & $18(4.5)$ & $>0.99$ \\
\hline $\begin{array}{l}\text { External Adnexa } \\
\text { Abnormalities }\end{array}$ & II (2.7) & $14(3.5)$ & 0.55 \\
\hline $\begin{array}{l}\text { Other Anterior } \\
\text { Segment } \\
\text { Abnormalities }\end{array}$ & $59(15)$ & $38(9.5)$ & $0.03 *$ \\
\hline
\end{tabular}

Notes: 'Between-treatment $p$-value comparing proportions based on Fisher's exact test. *Difference deemed significant at $p<0.05$.

Table 5 Symptom/Complaint Scores Over All Follow-Up Visits (Dispensed Eyes)

\begin{tabular}{|l|l|l|l|l|}
\hline \multirow{2}{*}{ Symptom/Complaint } & \multicolumn{3}{|l|}{ Mean Score \pm Sdev } \\
\cline { 2 - 5 } & $\mathbf{n}$ & Samfilcon A & $\mathbf{n}$ & Balafilcon A \\
\hline Burning/Stinging Upon & 400 & $95.8 \pm 7.3$ & 398 & $93.9 \pm 10.0$ \\
Insertion & & & & \\
Ease of Handling/Insertion & 400 & $95.9 \pm 7.7$ & 398 & $94.7 \pm 8.4$ \\
Vision Upon Insertion & 400 & $96.3 \pm 6.0$ & 398 & $94.5 \pm 8.7$ \\
Comfort Upon Insertion & 400 & $95.2 \pm 7.1$ & 398 & $92.2 \pm 10.3$ \\
Ease of Handling/Removal & 400 & $96.1 \pm 6.7$ & 399 & $94.9 \pm 8.1$ \\
Lens Cleanness Upon & 400 & $91.7 \pm 10.2$ & 399 & $88 \pm 13.3$ \\
Removal & & & & \\
Overall Comfort & 402 & $90.8 \pm 10.0$ & 400 & $86 \pm 14.2$ \\
Comfort at End of Day & 402 & $87.9 \pm 12.4$ & 400 & $83 \pm 16.3$ \\
Lens Handling & 400 & $95.7 \pm 7.4$ & 399 & $94.5 \pm 8.4$ \\
Dryness & 402 & $85.4 \pm 13.9$ & 400 & $80.1 \pm 16.9$ \\
Itchiness & 402 & $92.7 \pm 9.5$ & 400 & $91.3 \pm 11.9$ \\
Redness & 402 & $92.9 \pm 10.3$ & 400 & $93.5 \pm 9.8$ \\
Vision & 402 & $95.2 \pm 7.2$ & 400 & $92.4 \pm 10.0$ \\
Vision in Low Light & 402 & $94.6 \pm 7.6$ & 400 & $92.5 \pm 10.3$ \\
Vision at End of Day & 402 & $92.7 \pm 9.8$ & 400 & $90.2 \pm 11.9$ \\
Overall Impression & 402 & $91.3 \pm 9.9$ & 400 & $86.6 \pm 14.0$ \\
\hline
\end{tabular}

Notes: For each symptom, the score for each subject was obtained as follows: (I) the average score across OD and OS was calculated for each visit; (2) the mean of these per-visit averages was then calculated across all follow-up visits at which the subject presented data on the symptom.

\section{Discussion}

The objective of this study was to compare the respective performances of samfilcon A and balafilcon A contact lenses when worn for 7-days/6 nights of extended wear and replaced on a monthly basis.
Table 6 Subject-Wise Symptom/Complaint Unfavorable Findings Over All Follow-Up Visits

\begin{tabular}{|c|c|c|c|}
\hline \multirow[t]{2}{*}{ Symptom/Complaint } & \multicolumn{2}{|c|}{$\begin{array}{l}\text { Number/Total (Percent) of } \\
\text { Subjects Reporting an } \\
\text { Unfavorable Finding on } \\
\text { Either Eye on at Least One } \\
\text { Follow-up Visit }\end{array}$} & \multirow[t]{2}{*}{$\begin{array}{l}\mathrm{t}, \\
\text { *p-value }\end{array}$} \\
\hline & Samfilcon A & Balafilcon A & \\
\hline $\begin{array}{l}\text { Burning/Stinging Upon } \\
\text { Insertion }\end{array}$ & $17 / 400(4.3)$ & $28 / 398(7.0)$ & 0.09 \\
\hline Ease of Handling/Insertion & $15 / 400(3.8)$ & $12 / 398(3.0)$ & 0.70 \\
\hline Vision Upon Insertion & $9 / 400(2.3)$ & $12 / 398(3.0)$ & 0.52 \\
\hline Comfort Upon Insertion & $17 / 400(4.3)$ & $27 / 398(6.8)$ & 0.13 \\
\hline Ease of Handling/Removal & $1 \mathrm{I} / 400(2.8)$ & $16 / 399(4.0)$ & 0.34 \\
\hline $\begin{array}{l}\text { Lens Cleanness Upon } \\
\text { Removal }\end{array}$ & $23 / 400(5.8)$ & $47 / 399(12)$ & $0.003^{*}$ \\
\hline Overall Comfort & $42 / 402(10)$ & $74 / 400(19)$ & $0.00 I^{*}$ \\
\hline Comfort at End of Day & $48 / 402(12)$ & $81 / 400(20)$ & $0.002 *$ \\
\hline Lens Handling & $6 / 400(1.5)$ & $10 / 399(2.5)$ & 0.33 \\
\hline Dryness & $55 / 402(14)$ & $108 / 400(27)$ & $<0.000 I^{*}$ \\
\hline Itchiness & $49 / 402(12)$ & $53 / 400(13)$ & 0.67 \\
\hline Redness & $54 / 402(13)$ & $41 / 400(10)$ & 0.19 \\
\hline Vision & $23 / 402(5.7)$ & $38 / 400(9.5)$ & $0.046 *$ \\
\hline Vision in Low Light & $20 / 402(5.0)$ & $36 / 400(9.0)$ & $0.03 *$ \\
\hline Vision at End of Day & $25 / 402(6.2)$ & $41 / 400(10)$ & $0.04 *$ \\
\hline Overall Impression & $27 / 402(6.7)$ & $50 / 400(12)$ & $0.006 *$ \\
\hline
\end{tabular}

Notes: Unfavorable findings are defined as those with a reported score $<50$. The reported tally is based upon subjects that presented data for that symptom on at least one follow-up visit. ${ }^{\dagger}$ Between-treatment $\mathrm{p}$-value comparing proportions based on Fisher's exact test. *Difference deemed significant at $p<0.05$.

Slit lamp findings Grade 2 or greater were comparable for both lens types for all measured conditions except for corneal staining, which favored samfilcon A $(\mathrm{p}<0.05$; Table 3). Ungraded slit lamp findings were comparable for both lens types except for other anterior segment abnormalities, which favored balafilcon A ( $p<0.05$; Table 4).

Subjects rated both lenses highly with respect to vision and comfort (Table 5). The two lenses were comparable in most aspects but favored samfilcon A with respect to lens cleanliness upon removal, overall comfort, comfort at end of day, vision, vision in low light, vision at end of day, dryness, and overall impression (all $\mathrm{p}<0.05$; Table 6).

Balafilcon A lenses have been worn successfully on an extended wear basis for nearly two decades. Numerous clinical studies support the safety and effectiveness of the lens. Balafilcon A lenses worn in the same eye over 4 months on a 7-day extended wear basis and replaced weekly induced significantly less bulbar and limbal injection and corneal vascularization in 18 subjects than did 
etafilcon A lenses worn in the contralateral eye. ${ }^{26}$ Further, epithelial microcysts were observed in eyes that wore the etafilcon A lens, while no hypoxia-related events were observed in eyes that wore the balafilcon A lens.

In one study comparing traditional hydrogel to silicone hydrogel, the balafilcon A lens worn on a 30-day continuous wear basis in one eye performed better than did the etafilcon A lens worn on a 7-day extended wear schedule in the other eye based upon physiological parameters (epithelial microcysts, striae, corneal staining, limbal injection, and bulbar injection). ${ }^{27}$ Further, the study population rated the siliconehydrogel material superior to that of the traditional hydrogel in terms of dryness, comfort, lens handling, and overall subjective performance. In another study, complication rates in 504 patients randomized to 30-day continuous wear (353 patients) or to 7-day extended wear (151 patients) of balafilcon A lenses followed for 12 months were low, with no sight-threatening events or difference between 30-day and 7-day wear groups. ${ }^{28}$

Samfilcon A lenses were initially approved for daily wear in $2013,{ }^{3}$ and recently for 7-day/6-night extended wear; ${ }^{23}$ to our knowledge, this is the first study to report on the performance of the lens worn on an extended wear basis. However, forty samfilcon A wearers that wore the lens over a three month period and replaced the lens on a monthly basis rated the lens highly at all follow-up visits ( 2 weeks, 1 month, 2 months, and 3 months) in terms of overall comfort (range $89.1 \pm 11.7$ at 3 months to $91.1 \pm 13.0$ at 2 weeks on a $0-100$ point scale), vision $(92.0 \pm 12.6$ to $93.9 \pm 10.5)$, low-light vision $(90.4 \pm 12.9$ to $93.9 \pm 9.6)$, and cleanliness $(87.8 \pm 12.5$ to $91.0 \pm 9.8){ }^{22}$ Ratings were not different between two-week and one-month visits, nor between monthly visits. In contrast, a Johnson \& Johnson Vision Care-sponsored clinical study comparing samfilcon $\mathrm{A}$, senofilcon $\mathrm{C}$, comfilcon $\mathrm{A}$, and lotrafilcon $B$ lenses worn on a daily wear basis found that participants rated subjective overall comfort of samfilcon A (as well as comfilcon A and lotrafilcon B) lenses lower as wear time across the monthly replacement interval increased, ${ }^{29}$ contrary to our finding that samfilcon A lenses worn in 7-day extended wear mode maintain comfort over each weekly wear period, each monthly replacement period, and the full year duration of the study. As the former study is not published in a peer-reviewed journal, we have no explanation as to the discrepancy.

While samfilcon A lenses are approved for 7-day extended wear, balafilcon A and lotrafilcon B (Alcon, Ft. Worth, TX) are both approved for 30-day continuous wear. The latter two are reported to perform comparably within this mode with respect to lens fit, deposition and post-lens debris, burning/stinging, dryness, variation in vision, redness, and lens handling. ${ }^{30}$ However, the authors also reported that high contrast visual acuity and subjectreported quality of vision were statistically better with balafilcon A but not clinically significant. Further, for both lenses, limbal and conjunctival redness reduced throughout the duration of the study, whereas there was a slight increase in the overall amount of corneal staining.

\section{Study Limitations}

This study was designed to compare the performance of one silicone hydrogel lens recently cleared for EW in the US with one cleared decades ago over a 12-month period but not designed to determine the change in lens performance over the time course of the study. Thus, no inference can be drawn with respect to the stability of lens performance over time. The study also did not include other silicone hydrogel lenses cleared for either daily wear or EW. It would be interesting to compare such lenses from different manufacturers to determine which, if any particular lens characteristics (eg, material, design, packaging solution) contribute most to lens wear satisfaction.

\section{Conclusion}

Samfilcon A silicone hydrogel contact lenses perform comparable to or better than do balafilcon A lenses when worn on a 7-day extended wear basis, based upon the proportion of subjects with slit lamp findings Grade 2 or greater and ungraded slit lamp findings on either eye at any follow-up visit. In light of this finding, current wearers of samfilcon A lenses on a daily wear basis should expect a positive experience while wearing the lens on a 7-day extended wear basis.

\section{Funding}

We wish to thank Joseph Chinn for assistance in preparing this manuscript. This work was funded by Bausch \& Lomb Incorporated. This study is reported in the US National Library of Medicine database, with ClinicalTrials.gov Identifier NCT02939950. (Available from: https://www. clinicaltrials.gov/ct $2 /$ results ? cond $=\&$ term $=$ NCT02939950).

\section{Disclosure}

All authors but Howard Proskin are employed by Bausch \& Lomb Incorporated. Howard Proskin is paid consultant to Bausch \& Lomb Incorporated. The authors report no other conflicts of interest in this work. 


\section{References}

1. Morgan PB, Woods CA, Tranoudis IG, et al. International contact lens prescribing in 2019. Cont Lens Spectr. 2020;35:26-32.

2. FDA Premarket Approval (PMA) P040045. VISTAKON ${ }^{\circledR}$ (senofilcon A) contact lens, clear and visibility tinted with UV blocker; 2005. Available from: https://www.accessdata.fda.gov/cdrh_docs/pdf4/ p040045a.pdf. Accessed October 20, 2020.

3. FDA 510(k) Summary K131208. Bausch \& Lomb samfilcon A contact lens; 2013. Available from: https://www.accessdata.fda. gov/cdrh_docs/pdf13/K131208.pdf. Accessed October 20, 2020.

4. FDA Premarket Approval (PMA) P080011. BIOFINITY ${ }^{\circledR}$ (comfilcon A) soft contact lens for extended wear; 2008. Available from: https://www. accessdata.fda.gov/cdrh_docs/pdf8/P080011a.pdf. Accessed October 20, 2020.

5. FDA 510(k) Summary K073459. Lotrafilcon B Soft Contact Lenses; 2008. Available from: https://www.accessdata.fda.gov/cdrh_docs/ pdf7/K073459.pdf. Accessed October 20, 2020.

6. FDA Premarket Approval (PMA) P980006; Supplement 004. PureVision $^{\mathrm{TM}}$ (balafilcon A) visibility tinted contact lenses; 2001. Available from: https://www.accessdata.fda.gov/cdrh_docs/pdf/ P980006S004a.pdf. Accessed October 20, 2020.

7. FDA Premarket Approval (PMA) P010019; Focus Night and Day (lotrafilcon A) Soft Contact Lenses (extended wear for up to 30 nights of continuous wear); 2001. Available from: https://www.accessdata.fda. gov/cdrh_docs/pdf/P010019A.pdf. Accessed October 20, 2020.

8. Azuma $\bar{K}$, Ikeda K, Kagi N, Yanagi U, Osawa H. Evaluating prevalence and risk factors of building-related symptoms among office workers: seasonal characteristics of symptoms and psychosocial and physical environmental factors. Environ Health Prev Med. 2017;22 (1):38. doi:10.1186/s12199-017-0645-4

9. Sheppard AL, Wolffsohn JS. Digital eye strain: prevalence, measurement and amelioration. BMJ Open Ophthalmol. 2018;3(1):e000146. doi:10.1136/bmjophth-2018-000146

10. Jaiswal S, Asper L, Long J, Lee A, Harrison K, Golebiowski B. Ocular and visual discomfort associated with smartphones, tablets and computers: what we do and do not know. Clin Exp Optom. 2019;102(5):463-477. doi:10.1111/cxo.12851

11. Coles-Brennan C, Sulley A, Young G. Management of digital eye strain. Clin Exp Optom. 2019;102(1):18-29. doi:10.1111/cxo.12798

12. Cardona G, García C, Serés C, Vilaseca M, Gispets J. Blink rate, blink amplitude, and tear film integrity during dynamic visual display terminal tasks. Curr Eye Res. 2011;36(3):190-197. doi:10.3109/ 02713683.2010 .544442

13. Choi JH, Li Y, Kim SH, et al. The influences of smartphone use on the status of the tear film and ocular surface. PLoS One. 2018;13(10): e0206541. doi:10.1371/journal.pone.0206541

14. Willcox MDP, Argüeso P, Georgie GA, et al. TFOS DEWS II tear film report. Ocul Surf. 2017;15(3):366-403. doi:10.1016/j.jtos.2017.03.006

15. Ramamoorthy P, Nichols JJ. Compliance factors associated with contact lens-related dry eye. Eye Contact Lens. 2014;40(1):17-22. doi:10.1097/ICL.0000000000000009
16. Young G, Bowers R, Hall B, Port M. Six month clinical evaluation of a biomimetic hydrogel contact lens. CLAO J. 1997;23(4):226-236.

17. Schafer J, Reindel W, Steffen R, Mosehauer G, Chinn J. Use of a novel extended blink test to evaluate the performance of two polyvinylpyrrolidone-containing, silicone hydrogel contact lenses. Clin Ophthalmol. 2018;12:819-825. doi:10.2147/OPTH.S162233

18. Wygladacz K, Hook D, Steffen R, Reindel W. Breaking the cycle of discomfort. Cont Lens Spectr. 2014;29(13):23-28.

19. Hoteling AJ, Nichols WF, Harmon PS, et al. Characterization and quantitation of PVP content in a silicone hydrogel contact lens produced by dual-phase polymerization processing. $J$ Biomed Mater Res B. 2018;106B(3):1064-1072. doi:10.1002/jbm.b.33904

20. Steffen R, Merchea M, Rah M, Reindel W. Clinical performance of samfilcon A silicone hydrogel contact lenses. Cont Lens Spectr. 2014;29(13):30-38.

21. Reindel WT, Steffen R, Mosehauer G. Digital device users with dry eyes weigh in on a novel silicone hydrogel lens. Cont Lens Spectr. 2016;31(13):26-30.

22. Reindel W, Mosehauer G, Rah M, Steffen R. Performance of a unique silicone hydrogel lens across the recommended wear cycle. poster presentation, program number 165270, Annual Meeting of the American Academy of Optometry; Nov 17-20; 2010; San Francisco, CA, USA.

23. FDA Premarket Approval (PMA) P170035. Bausch + Lomb ULTRA (samfilcon A) contact lenses for extended wear; 2018. Available from: https://www.accessdata.fda.gov/cdrh_docs/pdf17/P170035a.pdf. Accessed October 20, 2020.

24. FDA Premarket Approval (PMA) P980006. PureVision ${ }^{\mathrm{TM}}$ (balafilcon A) visibility tinted contact lens for extended wear; 1999. Available from: https://www.accessdata.fda.gov/cdrh_docs/pdf/p980006.pdf. Accessed October 20, 2020.

25. FDA 510(k) Summary K083757. Bausch \& Lomb BPZO2 multipurpose solution; 2009. Available from: https://www.accessdata.fda. gov/cdrh_docs/pdf8/k083757.pdf. Accessed October 20, 2020.

26. Fonn D, MacDonald KE, Richter D, Pritchard N. The ocular response to extended wear of a high Dk silicone hydrogel contact lens. Clin Exp Optom. 2002;85(3):176-182. doi:10.1111/j.1444-0938.2002.tb03032.x

27. Brennan NA, Coles ML, Comstock TL, Levy B. A 1-year prospective clinical trial of balafilcon a (PureVision) silicone-hydrogel contact lenses used on a 30-day continuous wear schedule. Ophthalmology. 2002;109(6):1172-1177. doi:10.1016/S0161-6420(02)01045-X

28. Nilsson SE. Seven-day extended wear and 30-day continuous wear of high oxygen transmissibility soft silicone hydrogel contact lenses: a randomized 1-year study of 504 patients. CLAO J. 2001;27:125-136.

29. Johnson \& Johnson Vision Care, Inc. Clinical study of approved and investigational contact lenses. ClinicalTrials.gov identifier NCT02708524; 2016. Available from: https://clinicaltrials.gov/ct2/ show/study/NCT02708524. Accessed October 20, 2020.

30. Morgan PB, Efron N. Comparative clinical performance of two silicone hydrogel contact lenses for continuous wear. Clin Exp Optom. 2002;85 (3):183-192. doi:10.1111/j.1444-0938.2002.tb03033.x
Clinical Ophthalmology

\section{Publish your work in this journal}

Clinical Ophthalmology is an international, peer-reviewed journal covering all subspecialties within ophthalmology. Key topics include: Optometry; Visual science; Pharmacology and drug therapy in eye diseases; Basic Sciences; Primary and Secondary eye care; Patient Safety and Quality of Care Improvements. This journal is indexed on PubMed
Central and CAS, and is the official journal of The Society of Clinical Ophthalmology (SCO). The manuscript management system is completely online and includes a very quick and fair peer-review system, which is all easy to use. Visit http://www.dovepress.com/ testimonials.php to read real quotes from published authors.

\section{Dovepress}

